\title{
Assessment of Mixtures by Spectral Superposition. An Approach for the Field of Metabolomics
}

René Bachmann ${ }^{1}$, Navid Shakiba ${ }^{2}$, Markus Fischer $^{2}$ and Thomas Hackl ${ }^{1,2 *}$

${ }^{1}$ Institute of Organic Chemistry, University of Hamburg, Martin-Luther-King-Platz 6, 20146

Hamburg, Germany *Corresponding author: Tel.: +49-40-428382804 E-Mail:

Thomas.Hackl@chemie.uni-hamburg.de

${ }^{2}$ Hamburg School of Food Science; Institute of Food Chemistry, University of Hamburg, Grindelallee 117, 20146 Hamburg, Germany

Table of Contents

Supplemental Material Table S-1

Supplemental Material Table S-2

Supplemental Material Figure S-1

Supplemental Material Figure S-2

Supplemental Material Figure S-3

Supplemental Material Figure S-4
Table listing information of used hazelnut samples

Detailed list of used buckets

Comparison of signal deviation for two different buckets

Supplementary confusion matrices for the twoclass classfier for Italy, France and Germany

Corresponding ROC-AUC curves for all used classifers

Bar chart displaying the deviation of calculated and measured samples 
Table S1: Information about origin, variety and supplier for the authentic hazelnut samples using for mixture analysis

${ }^{1}$ EO: Erzeugerorganisation

\begin{tabular}{|c|c|c|c|c|c|}
\hline supplier & $\begin{array}{c}\text { harvest } \\
\text { year }\end{array}$ & Variety & country & $\begin{array}{l}\text { declared } \\
\text { region }\end{array}$ & $\begin{array}{c}\text { declared } \\
\text { origin }\end{array}$ \\
\hline $\begin{array}{c}\text { AgroTeamConsulting/NocciolaR } \\
\mathrm{e}\end{array}$ & 2015 & $\begin{array}{l}\text { Mortarell } \\
\text { a }\end{array}$ & Italy & Kampanien & Teano \\
\hline $\begin{array}{l}\text { STELLIFERI \& ITAVEX s.r.l. } \\
\text { Unipersonale }\end{array}$ & 2015 & $\begin{array}{l}\text { Tonda } \\
\text { Gentile } \\
\text { Trilobata }\end{array}$ & Italy & Piemont & Alba \\
\hline $\begin{array}{l}\text { AgroTeamConsulting/ } \\
\text { NocciolaRe }\end{array}$ & 2016 & $\begin{array}{l}\text { Tonda } \\
\text { (Gentile) } \\
\text { Romana }\end{array}$ & Italy & Latium & Vallerano \\
\hline $\begin{array}{l}\text { EO Deutscher Haselnussanbauer } \\
\text { UG }^{1}\end{array}$ & 2015 & mixture & Germany & Bavaria & Pförring \\
\hline $\begin{array}{c}\text { EO Deutscher Haselnussanbauer } \\
\text { UG }^{1}\end{array}$ & 2015 & mixture & Germany & $\begin{array}{l}\text { Rhineland } \\
\text { Palatinate }\end{array}$ & Nieder-Wiesen \\
\hline $\begin{array}{l}\text { EO Deutscher Haselnussanbauer } \\
\text { UG }^{1}\end{array}$ & 2014 & mixture & Germany & Bavaria & Burgheim \\
\hline Ferrero OHG mbh & 2015 & mixture & Turkey & Düzce & Akcakoca \\
\hline Ferrero OHG mbh & 2016 & unknown & Turkey & unknown & unknown \\
\hline Heinrich Brüning GmbH & 2014 & mixture & Georgia & unknown & unknown \\
\hline $\begin{array}{c}\text { Lübecker Marzipan-Fabrik v. } \\
\text { Minden \& Bruhns GmbH \& Co. } \\
\text { KG }\end{array}$ & 2015 & mixture & Georgia & unknown & unknown \\
\hline SCA Unicoque & 2014 & Corabel & France & $\begin{array}{c}\text { Poitou- } \\
\text { Charentes }\end{array}$ & Vienne \\
\hline SCA Unicoque & 2015 & Pauetet & France & Aquitanien & Dordogne \\
\hline SCA Unicoque & 2016 & Butler & France & $\begin{array}{c}\text { Midi- } \\
\text { Pyrénées }\end{array}$ & $\begin{array}{l}\text { Tarn-et- } \\
\text { Garonne }\end{array}$ \\
\hline $\begin{array}{c}\text { Schlüter \& Maack } \\
\text { GmbH/Hanseatic Trading Blaase } \\
\text { GmbH } \\
\end{array}$ & 2014 & Tombul & Turkey & Ordu & Ordu \\
\hline $\begin{array}{c}\text { Schlüter \& Maack } \\
\text { GmbH/Hanseatic Trading Blaase } \\
\text { GmbH }\end{array}$ & 2015 & Megrelia & Georgia & $\begin{array}{l}\text { Mingrelien } \\
\text { and } \\
\text { Oberswanetie } \\
\mathrm{n}\end{array}$ & Zugdidi \\
\hline
\end{tabular}


Table S2: Variable sized buckets

[ppm]: 0.8867-0.8800; 0.8957-0.8880; 0.8970-0.9063; 0.9070-0.9147; 0.9147-0.9182; 0.9182-0.9246; 0.9320-0.9249; $0.9432-0.9503 ; 0.9509-0.9603 ; 0.9609-0.9769 ; 0.9853-0.9795 ; 0.9927-0.9856 ; 1.0013-1.0123 ; 1.0209-1.0299 ; 1.0347-$ $1.0472 ; 1.0530-1.0636 ; 1.1313-1.1554 ; 1.2161-1.2434 ; 1.3137-1.3467 ; 1.4649-1.5008 ; 1.8347-1.8462 ; 1.8934-1.8504$; $1.9037-1.9162 ; 1.9284-1.9431 ; 1.9444-1.9560 ; 1.9579-1.9708 ; 1.9714-1.9891 ; 1.9897-2.0038 ; 2.0048-2.0234 ; 2.0240-$ $2.0301 ; 2.0311-2.0443 ; 2.0491-2.0616 ; 2.0635-2.0680 ; 2.0680-2.0796 ; 2.0805-2.0873 ; 2.0873-2.0953 ; 2.0982-2.1126$; $2.1171-2.1313 ; 2.1364-2.1499 ; 2.1531-2.1688 ; 2.1730-2.1797 ; 2.2430-2.2593 ; 2.2600-2.2683 ; 2.2686-2.2776$; $2.2783-$ $2.2850 ; 2.2866-2.2953 ; 2.2959-2.3030 ; 2.3139-2.3251 ; 2.3341-2.3405 ; 2.3409-2.3482 ; 2.3521-2.3630 ; 2.3704-2.3749$; $2.3749-2.3781 ; 2.3781-2.3861 ; 2.3864-2.3913 ; 2.3935-2.3999 ; 2.4003-2.4054 ; 2.4198-2.4295 ; 2.4802-2.5081 ; 2.5091-$ $2.5206 ; 2.5213-2.5287 ; 2.5415-2.5447 ; 2.5312-2.5412 ; 2.5466-2.5518 ; 2.5547-2.5640 ; 2.5755-2.5819 ; 2.5909-2.5996$; 2.6310-2.6904; 2.6266-2.6310; 2.6917-2.6994; 2.7097-2.7155; 2.7437-2.7553; 2.7733-2.7906; 2.7960-2.8034; 2.8172$2.8236 ; 2.8272-2.8323 ; 2.8326-2.8362 ; 2.8368-2.8426 ; 2.8439-2.8493 ; 2.8509-2.8602 ; 2.8638-2.8712 ; 2.8718-2.8795$; $2.8801-2.8850 ; 2.9017-2.9097 ; 2.9174-2.9248 ; 2.9277-2.9334 ; 2.9341-2.9398 ; 2.9402-2.9453 ; 2.9594-2.9694 ; 2.9710-$ $2.9803 ; 2.9858-2.9928 ; 2.9976-3.0134 ; 3.0201-3.0307 ; 3.0358-3.0455 ; 3.0461-3.0589 ; 3.0647-3.0753 ; 3.0760-3.0869$; $3.0978-3.1106 ; 3.1148-3.1196 ; 3.1202-3.1286 ; 3.1292-3.1331 ; 3.1334-3.1373 ; 3.1379-3.1479 ; 3.1540-3.1645 ; 3.1710-$ $3.1793 ; 3.1828-3.1931 ; 3.1992-3.2165 ; 3.2191-3.2265 ; 3.2278-3.2435 ; 3.2454-3.2519 ; 3.2525-3.2596 ; 3.2605-3.2763$; $3.2933-3.3023 ; 3.3385-3.3433 ; 3.3453-3.3520 ; 3.3523-3.3607 ; 3.3613-3.3697 ; 3.3700-3.3790 ; 3.3796-3.3905 ; 3.3912-$ $3.4027 ; 3.4034-3.4133 ; 3.4278-3.4859 ; 3.4904-3.4984 ; 3.5032-3.5103 ; 3.5154-3.5337 ; 3.5356-3.5392 ; 3.5395-3.5619$; $3.5706-3.5764 ; 3.5770-3.5838 ; 3.5873-3.6027 ; 3.6184-3.6255 ; 3.6287-3.6338 ; 3.6342-3.6438 ; 3.6477-3.6531 ; 3.6550-$ $3.6605 ; 3.6615-3.6833 ; 3.6997-3.7074 ; 3.7118-3.7208 ; 3.7240-3.7420 ; 3.7449-3.7523 ; 3.7642-3.7706 ; 3.7529-3.7642$ : $3.7754-3.7882 ; 3.7982-3.8139 ; 3.8152-3.8229 ; 3.8232-3.8277 ; 3.8281-3.8329 ; 3.8332-3.8393 ; 3.8399-3.8463 ; 3.8467-$ $3.8553 ; 3.8557-3.8653 ; 3.8659-3.8756 ; 3.8759-3.8897 ; 3.8900-3.8996 ; 3.9536-3.9581 ; 3.9382-3.9523 ; 3.9719-3.9902$; 3.9905-4.0004; 4.0123-4.0727; 4.0746-4.0823; 4.0887-4.0961; 4.0999-4.1192; 4.1230-4.1349; 4.1449-4.1600; 4.1632$4.1757 ; 4.1978-4.2017 ; 4.1808-4.1969 ; 4.2049-4.2174 ; 4.2197-4.2242 ; 4.2306-4.2376 ; 4.2392-4.2460 ; 4.2585-4.2752$; 4.2845-4.2999; 4.3051-4.3131; 4.3166-4.3266; 4.3625-4.3718; 4.3859-4.3936; 4.4200-4.4296; 4.4412-4.4498; 4.60264.6447; 4.9721-4.9968; 5.0109-5.0215; 5.1846-5.2244; 5.3923-5.4260; 5.4263-5.4356; 5.5964-5.6003; 5.6016-5.6057; $5.6067-5.6176 ; 5.6199-5.6269 ; 5.8741-5.8828 ; 5.8940-5.9052 ; 5.9104-5.9187 ; 6.5103-6.5167 ; 6.8021-6.8162 ; 6.8323-$ $6.8519 ; 6.8702-6.8814 ; 6.8872-6.9000 ; 6.9010-6.9270 ; 6.9822-6.9937 ; 7.0021-7.0149 ; 7.0798-7.0881 ; 7.1019-7.1131$; 7.1212-7.1337; 7.1420-7.1517; 7.1770-7.1870; 7.1966-7.2059; 7.2582-7.2714; 7.3131-7.3253; 7.3324-7.3536; 7.36227.3719; 7.3754-7.3918; 7.3976-7.4101; 7.4146-7.4264; 7.4839-7.4938; 7.5032-7.5144; 7.7108-7.7198; 7.8813-7.8868; 7.9018-7.9073; 8.3525-8.3596; 8.4562-8.4620;8.8356-8.8504; 8.8533-8.8696; 9.1258-9.1444 

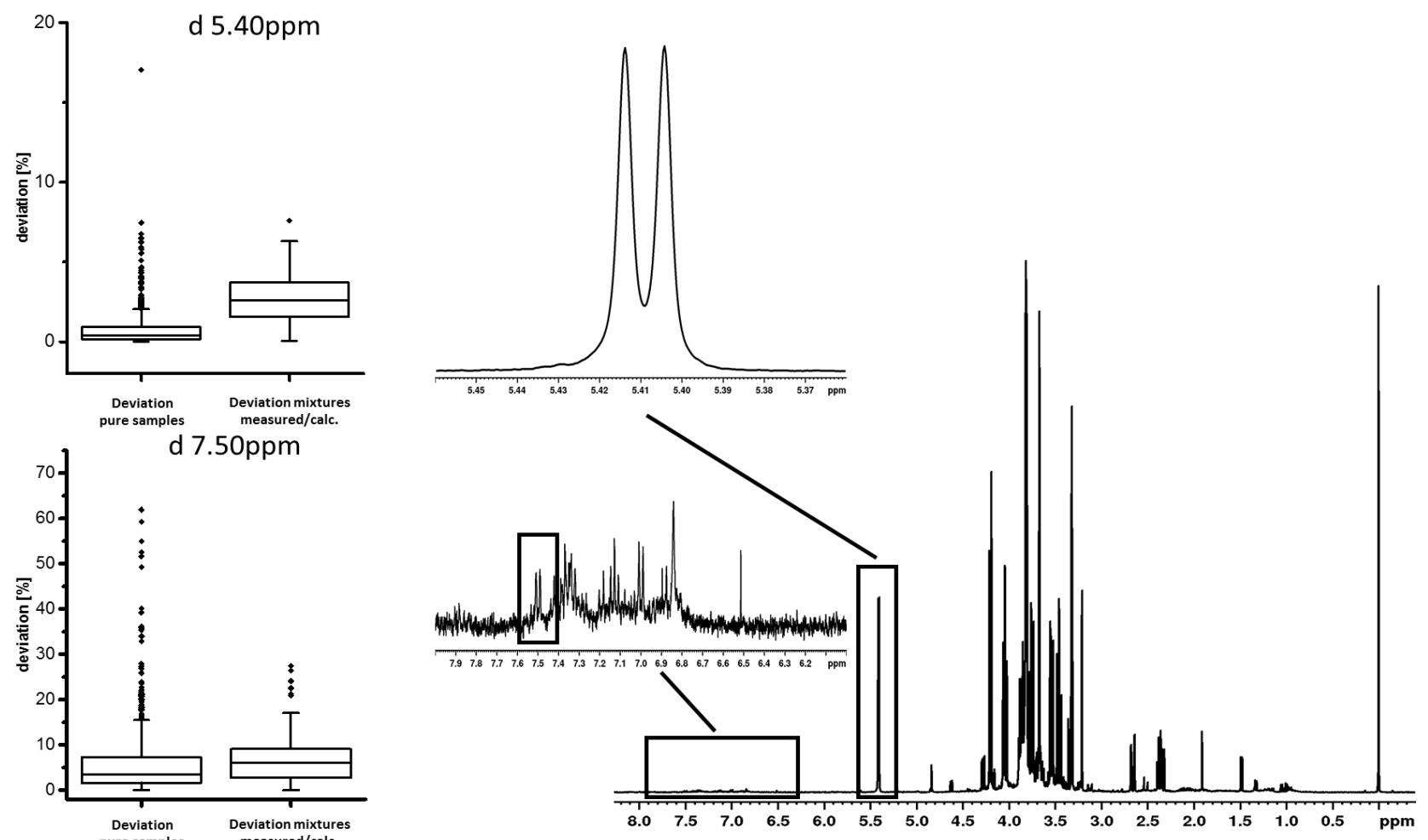

Figure S1: Comparison of signal deviation. Due to a missing standard for quality estimation, the deviations from calculated to measured spectra were compared with the deviations of the triple determinations of the pure samples. As expected, the variances in both groups increases with decreasing metabolite concentrations. The deviations of the calculated to measured spectra are always a few percent higher as for the pure samples, but similar enough for the use in a classifier.
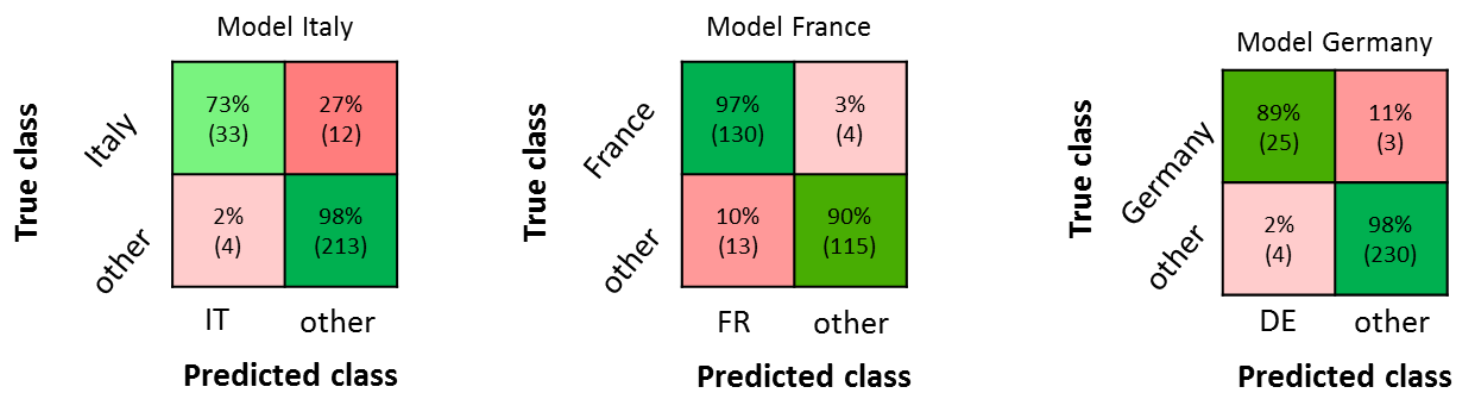

Figure S2: Confusion Matrices for the two-class Classifier for Italy, France and Germany 

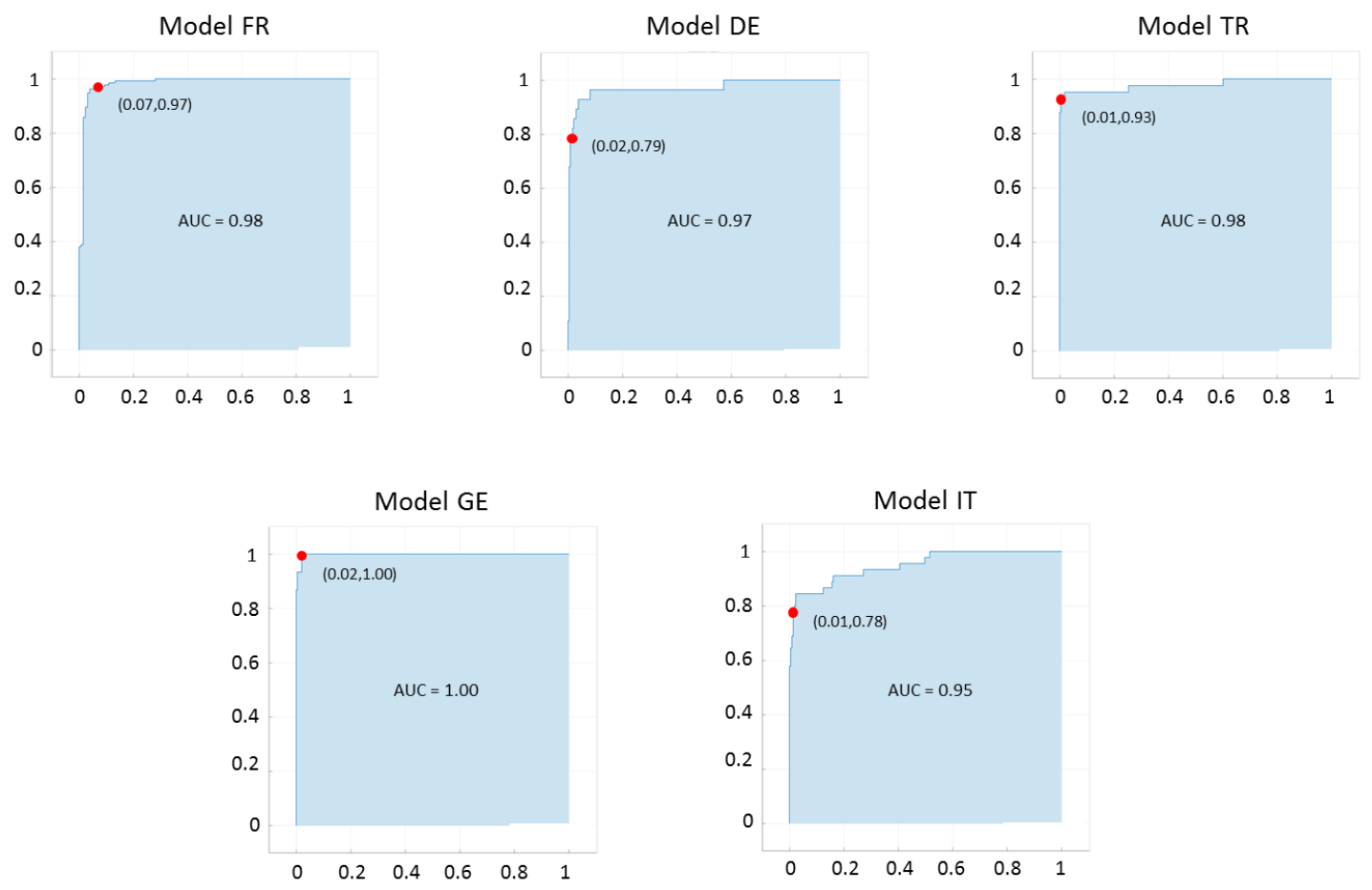

Figure S3: Respective ROC-AUC-curves for the two-class Classifier 


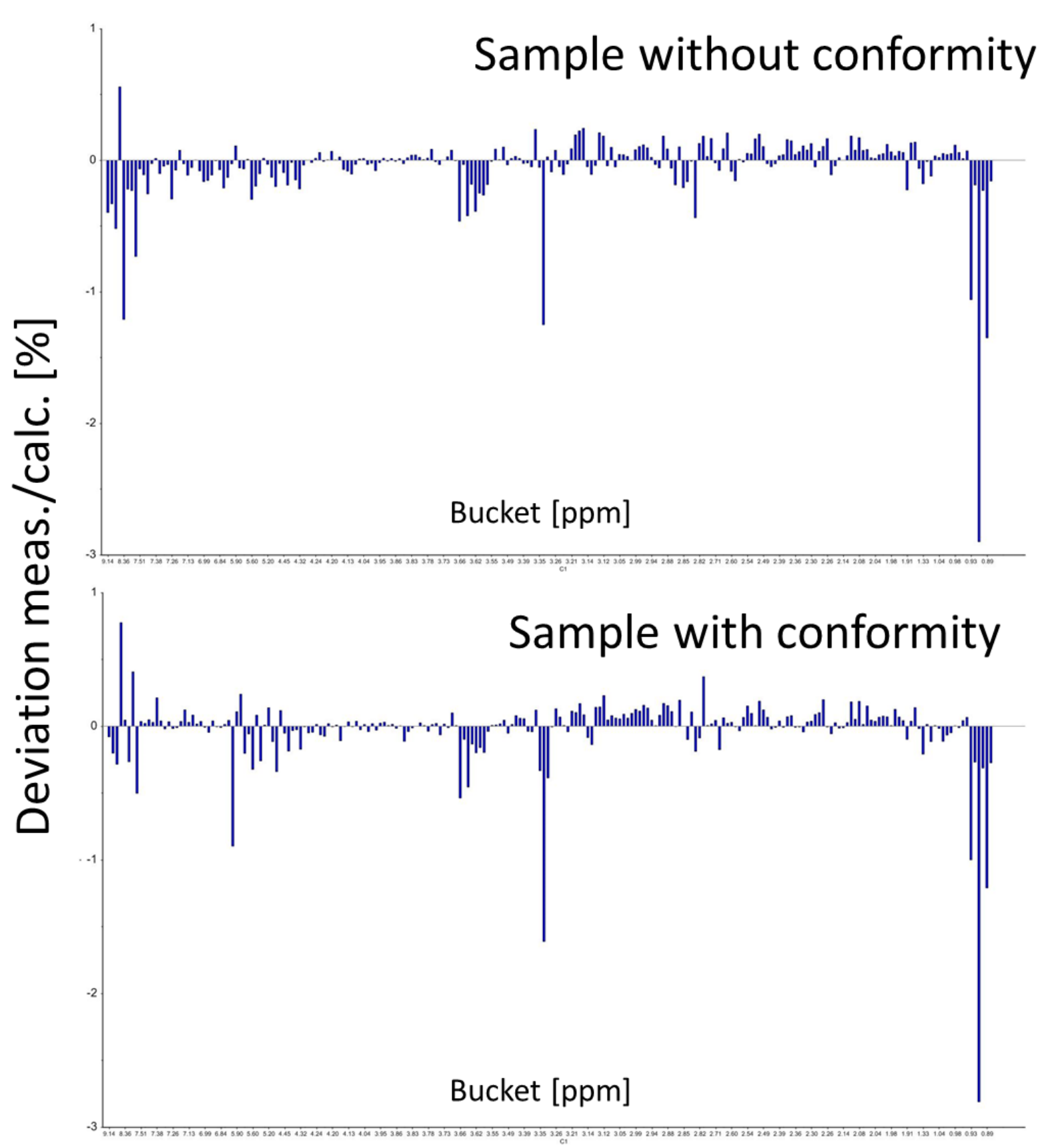

Figure S4: Full bar plot of the deviations of calculated and measured spectra for samples with and without conformity in the classification model. For both, the deviation of buckets with low $\mathrm{S} / \mathrm{N}$ is higher compared to higher concentrated signals. The samples differ mainly in these buckets. 